\title{
Posterior sternoclavicular dislocation: an American football injury
}

Department of Orthopaedics, Hvidovre Hospital, Copenhagen, Denmark

L B Marker

B Klareskov

Correspondence to: Dr L Marker,

Department of Orthopaedics, Køge Sygehus, 4600 Køge, Denmark.

Accepted for publication 7 June 1995

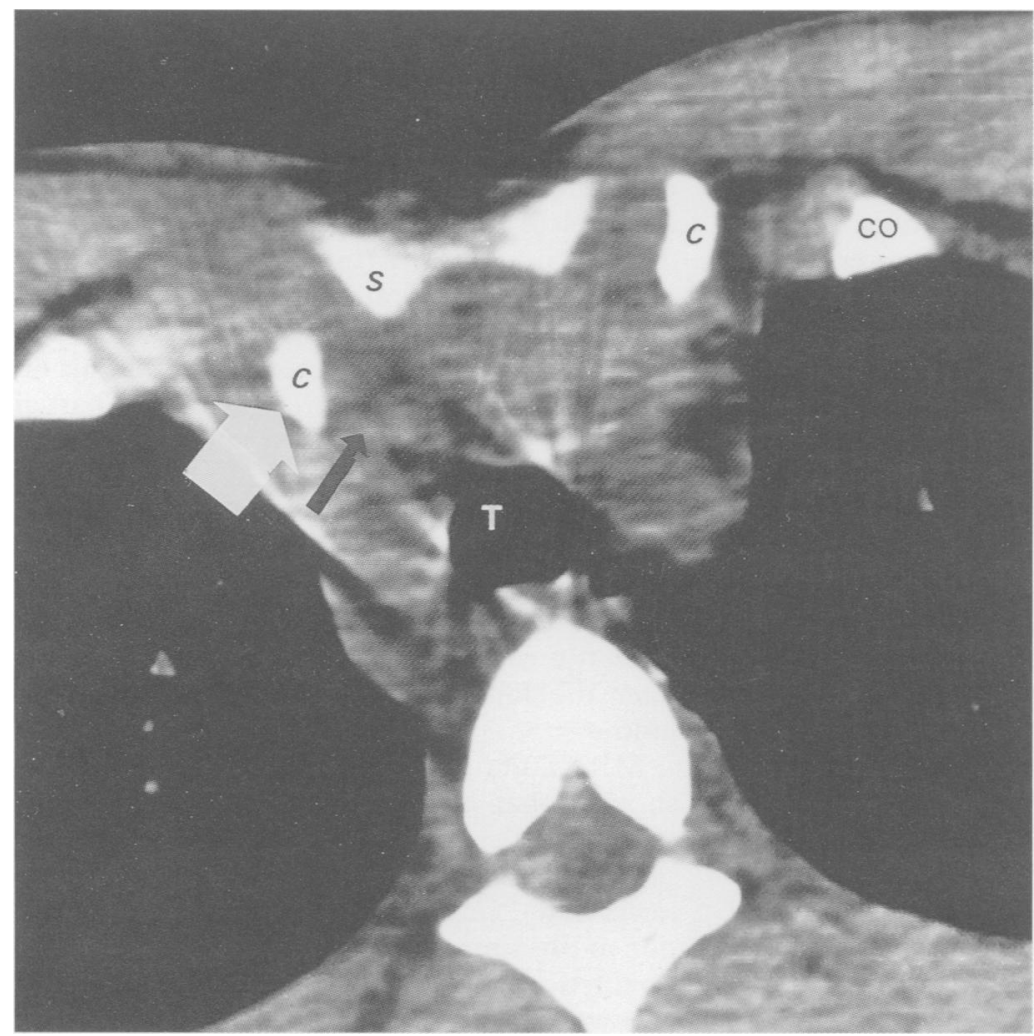

Computerised tomographic scan showing posterior sternoclavicular dislocation and mediastinal haematoma related to the left subclavian vein. palpable depression of the medial part of the right clavicle. Radiological examination showed posterior sternoclavicular dislocation. On computerised tomography (CT), there was a mediastinal haematoma related to the right sternoclavicular joint (figure). Injection of $x$ ray contrast fluid showed no damage or compression of the right subclavian vein. Reduction under local anaesthesia did not succeed. Under general anaesthesia the patient was placed supine with a sandbag between the scapulae. Simultaneous postero-lateral traction of the upper arm and digital traction of the medial clavicle forwards reduced the joint. Control CT scan confirmed the reduction. A figure of eight bandage was applied for six weeks. At follow up the patient had no symptoms and full range of motion.

\section{Discussion}

The sternoclavicular joint is stabilised by a solid joint capsule and several ligaments, and the fact that the posterior sternoclavicular ligament is the strongest probably makes the dorsal dislocation far more uncommon than anterior dislocation. ${ }^{1-5}$ The mobility and laxity of the joint decreases with age, and for this reason most of the reported cases occur in young people, mainly males, and the mechanism is a violent blow on the postero-lateral aspect of the shoulder with the arm adducted and flexed. ${ }^{8}$ This position of the arm is typical in American football when a player holds the ball and tries to avoid his opponents. During a tackle the shoulder often is unprotected and exposed to injury.

Several reported injuries have also been sustained by young males involved in motorcycle accidents. ${ }^{7}$ Typically these patients present with the neck flexed towards the injured side, supporting the flexed elbow with the opposite hand and complaining of local pain. ${ }^{3} 7$ A depression can be seen and palpated if swelling has not yet obscured the the picture. To confirm the diagnosis, oblique $x$ rays are recommended, since a routine $\mathrm{AP}$ view often does not show the condition. ${ }^{6}$ Because of the close relation of the posteriorly displaced medial clavicle to several vital structures, serious complications have been reported. Pneumothorax and haemothorax, and compression or even laceration of the great vessels or the trachea have been seen. ${ }^{7}$ Where the condition has gone unrecognised thoracic outlet syndrome has been described. Further investigations are therefore necessary to rule out any life threatening injuries to these structures, including plain films of the chest, a 
CT scan, and angiography if any involvement of the vessels is suspected.

Stable closed reduction of a posterior sternoclavicular dislocation can be done successfully using the method described in our case. A towel clip to grip the clavicle medially has been found to be helpful. Open reduction may be necessary with repair or fixation by one of various methods described. ${ }^{27}$ Transfixion with $\mathrm{K}$ wires is not recommended because of the risk of migration and potential damage to vital organs. ${ }^{1}$ Resection of the medial part of the clavicle may be the best choice, especially in cases discovered late. A figure of eight bandage for six weeks is sufficient for after care.

Although posterior sternoclavicular dislocations are rare, the potential serious com- plications makes early diagnosis and treatment important.

1 Gangahar D, Flogaites T. Retrosternal dislocation of the clavicle producing thoracic outlet syndrome. F Trauma 1978;18:369-72.

2 Lewonowski K, Bassett GS. Complete posterior sternoclavicular epiphyseal separation. Clin Orthop 1992;281: 84-8.

3 Gazak S, Davidson SJ. Posterior sternoclavicular dislocations: two case reports. $\mathcal{F}$ Trauma 1984;24:80-2.

4 Paterson D. Retrosternal dislocation of the clavicle. $\mathcal{f}$ Bone foint Surg 1961;43:90-4.

5 Leighton RK, Buhr AJ. Posterior sternoclavicular dislocations. Can f Surg 1986;29:104-6.

6 Wehner. Zur Konservation behandlung der Retrosternale Luxation der Klavikular Luxation. F Ortho Chir 1931;55: 409.

7 Worman LW, Leagus C. Intrathoracic injury following retrosternal dislocation of the clavicle. $\mathcal{F}$ Trauma 1967;7: retrosternal

8 Greelee DP. Posterior dislocation of the sternal end of the clavicle. f Am Med 1944; June:426-8.

\title{
Complete avulsion of the hamstring tendons from the ischial tuberosity. A report of two cases sustained in judo
}

\author{
Hideki Kurosawa, Ken Nakasita, Hiroyasu Nakasita, Shigeru Sasaki, Seigou Takeda
}

\begin{abstract}
Rupture of the hamstring tendon is a rare injury. Two cases of complete rupture of the hamstring tendons sustained while playing judo are reported. The diagnosis of a rupture of the hamstring tendons was difficult from physical examination because of severe pain on knee or hip joint movement. Magnetic resonance imaging was non-invasive and showed the lesion clearly. In one of these two cases the less satisfactory results of non-operative treatment were clearly shown in both isokinetic muscle force evaluation and sports activities.

(Br F Sports Med 1996;30:72-74)
\end{abstract}

Key terms: hamstring, avulsion, ischial tuberosity, judo.

Rupture of the hamstring tendon is a rare injury in sporting activities. ${ }^{1-3}$ We report two cases of complete rupture of the hamstring tendons sustained while playing judo. In neither case was a fracture of the ischial tuberosity identified on $x$ ray. One case was treated nonoperatively and the other operatively.

\section{Case report}

CASE 1

A 28 year old male, Judo 4 dan (fourth grade judo player), felt severe pain in the posterior side of the thigh of the pivoting leg when he was about to do the uchimata (inner thigh) move on his opponent during training (fig 1). It became impossible for him to stand on the leg and could not walk because of severe pain. He visited our outpatient clinic on the next day. On physical examination, no clearly palpable hollow indicating rupture of the hamstring tendons or muscles was discernible because of marked swelling and widespread subcutaneous bleeding; there was severe tenderness and pain on motion of the hip or knee joint. Magnetic resonance imaging (MRI) evaluation revealed a complete rupture of hamstring tendon from the ischial tuberosity (fig 2 , upper row).

$\mathrm{He}$ refused operative treatment and was immobilised in a single spica cast with the hip joint in neutral and the knee joint at about 60 degrees of flexion for three weeks. A partial weight bearing gait was then started. A range of motion exercises for the joints and stretching exercises of the muscles was begun as the pain lessened. Strengthening exercises were also introduced as for a strain of the hamstring. ${ }^{4} \mathrm{He}$ could walk with full weight bearing six weeks after the injury without support. Ten weeks after the injury, he resumed his training in judo.

MRI findings seven years after the injury showed that the ruptured end of the hamstring muscles remained within the soft tissues separate from the ischial tuberosity (fig 2, lower row). In the evaluation of muscle function at this time (Myoret, Kawasaki Jukoh Co), isokinetic hamstring strength on the injured side was $20-40 \%$ less than on the non-injured side, while the quadriceps power was equal on both sides. 